Apidologie, 1975, 6 (3), 195-206.

\title{
DAS POLLENBILD EINIGER NIEDERLÄNDISCHER HONIGE
}

\author{
Spectre pollinique de quelques miels néerlandais
}

\author{
J. D. KERKVLIET und A.P.J. VAN DER PUTTEN
}

Keuringsdienst van waren (Lebensmitteluntersuchungsamt) Haarlem, Niederlande*

\section{SUMMARY}

POLLEN SPECTRUM OF SOME DUTCH HONEYS

A number of authentic Dutch honey samples, obtained trough the kind cooperation of various bee-keepers was microscopically examined on the presence of pollen, in order to get an impression of the pollen spectrum of Dutch honey samples. Also the relation between the nectar source of the honey, as obtained from the observations by bee-keepers, and the microscopical analysis was verified. In this connection e.g. the percentage Tilia (Lime) pollen in the honey samples, which were specified as lime honey by bee-keepers, was determined. Also, in heather (Calluna) honey, the amount of Calluna and Erica pollen was investigated, in combination with the results of the thixotropytest according to Louveaux. Previously DE BoER had investigated the pollen spectrum of Dutch honey samples; in this study a comparison is made between the present and the former observations. Summarizing one can say that in Dutch honeys Trifolium repens (white clover) is present, in combination with Tilia (lime), Brassica napus (rape), Compositeae (composites), Ligustrum (privet), Salix (willow) and Prunus (fruit trees). For Dutch heather honey the combination of Trifolium repens, Calluna vulgaris (common ling), Fagopyrum esculentum (buckwheat), Castanea (sweet chesnut) Erica tetralix (bell-heather), Centaurea cyanus (cornflower) and Helianthus (sunflower) is characteristic.

\section{ZUSAMMENFASSUNG}

Eine Anzahl authentischer niederländischer Honigproben, welche uns durch die freundliche Mitwirkung verschiedener Imker zur Verfügung gestellt worden sind, wurden mikroskopisch auf das Vorkommen von Pollen untersucht, um einen Eindruck vom Pollenspektrum des niederländischen Honigs zu bekommen.

Daneben wurde geprüft, inwieweit die Sortenbezeichung auf Grund der imkerlichen Beobachtungen mit dem mikroskopischen Befund übereinstimmt.

* Nieuwe Gracht 3, Haarlem/Niederlande. 
In diesem Zusammenhang wurde der Prozentsatz der Tilia-Pollen in von Imkern als Lindenblütenhonig bezeichneten Proben bestimmt und wurde für Heidehonig sowohl die Untersuchung des Gehaltes an Calluna- und Erica-Pollen vorgenommen als auch der Thixotropie-Test nach LouvEAUX durchgeführt.

Geprüft wurde ausserdem, inwieweit das Pollenspektrum mit den fräheren von DE BoER durchgeführten Untersuchungen von niederländischem Honig übereinstimmt.

Zusammenfassend kann gesagt werden, dass in niederländischen Honigen Trifolium repens auftritt, in Kombinationen mit Tilia, Brassica napus, Compositeae, Ligustrum, Salix und Prunus.

Für niederländischen Heidehonig ist die Kombination Trifolium repens, Calluna vulgaris, Fagopyrum esculentum, Castanea, Erica tetralix, Centaurea cyanus und Helianthus charakteristisch.

\section{EINFÜHRUNG}

Die mikroskopische Honiguntersuchung hat den Zweck, die geographische und botanische Herkunft eines Honigs festzustellen.

Nach der EWG-Verordnung soll ein als Sortenhonig bezeichnetes Produkt vorwiegend von der betreffenden Trachtquelle stammen. Eine derartige Bestimmung gibt es auch in der niederländischen Honigverordnung; eine Methode, um die botanische Herkunft festzustellen, wird nicht vorgeschriehen. Es wird nur angegeben, dass man diese Bestimmung mit Hilfe der Pollenanalyse macht.

Da es im Gebiet des Haarlemmer Lebensmitteluntersuchungsamtes eine Anzahl grosser Honigimportfirmen gibt, ist die Frage nach der richtigen botanischen Bezeichnung für uns wichtig. Wir haben das Pollenbild stets nach der Arbeitsvorschrift, wie sie von der Internationalen Kommission für Bienenbotanik der I.U.B.S. publiziert ist (Louveaux, Madrizio, Vorwohl 1970), interpretiert, es sei denn, dass für Lindenblütenhonig und einige andere Sorten mit unterrepräsentierten Pollen unsere Normen etwas niedriger gewählt sind.

In der niederländischen Honigverordnung (Artikel 2) wird auch ein Unterschied zwischen inländischem und ausländischem Honig gemacht.

Ein Honig soll dann als ausländisch bezeichnet werden, wenn er nicht aus dem Benelux-Gebiet stammt, oder wenn es sich um eine Mischung inländischen und ausländischen Honigs handelt.

In der Praxis fehlt zuweilen auf Importhonig die Bezeichnung “ Ausländischer Honig 》. Dadurch wird der Eindruck erweckt, dass es sich um einen Benelux-Honig handelt. Ein derartiges Verfahren kann nur mit Hilfe der Pollenanalyse erkannt werden.

Vor mehreren Jahren publizierte DE BoER schon das Pollenspektrum von niederländischen Honigen (DE BoEr 1957). Der Zweck unserer Untersuchungen war, festzustellen, welche Pollenformen jetzt in niederländischen Honigen vorherrschen und von welchen Formen sie begleitet werden. 
Dazu wurden, durch die freundliche Mitwirkung verschiedener Imker, viele Proben reiner niederländischer Honige gesammelt und mikroskopisch untersucht.

Daneben wurde geprüft, inwieweit die Sortenbezeichnung auf Grund der imkerlichen Beobachtungen mit dem mikroskopischen Befund übereinstimmt.

\section{MATERIAL UND METHODEN}

Insgesammt wurden 41 Honigproben untersucht. Sie stammten hauptsächlich aus 1971 und 1972 und kamen aus dem ganzen Lande, obwohl die Mehrzahl der Frühjahrs- und Lindenblütenhonige aus der Umgebung von Haarlem, also aus dem westlichen Dünengebiet stammte.

Die Anfertigung der Honigpräparate und die Auswertung erfolgte nach den Angaben der Internationalen Kommission für Bienenbotanik (Louveaux, Maurizio, und VorwoHL 1970). Daneben wurde für Heidehonige auch der Thixotropie-Test nach Louveaux durchgeführt (Louveaux 1966 und 1968).

Es sei noch darauf hingewiesen, dass diese Arbeit in kurzer Form bereits in einer niederländischen Zeitschrift veröffentlicht wurde (Kenkvliet, Van Der Putten, 1973).

\section{RESULTATE}

\section{Pollenanalyse der verschiedenen Honige}

Die Untersuchungsresultate der einzelnen Honigproben sind in Tabelle 1 angegeben; in dieser Tabelle sind die Pflanzennamen nach Familien geordnet (nach Engler 1964). Die identifizierten Pollenformen wurden in Häufigkeitsklassen eingeteilt, nach Angabe der Internationalen Kommission für Bienenbotanik. Die deutschen und niederländischen Namen der gefundenen Pflanzenarten sind aus der Tabelle auf s. 206 zu entnehmen.

Die untersuchten Honigproben sind in fünf Gruppen geordnet; die ersten 4 Gruppen stehen in Zusammenhang mit dem Zeitpunkt der Ernte, nämlich Frühjahrshonig, Lindenblütenhonig, Sommerhonig und Heidehonig (Spättracht).

Dabei sind auch die imkerlichen Angaben über die mutmassliche Herkunft aufgenommen.

Die fünfte Gruppe umfasst einige Spezialtrachten wie Raps-, Strandasterund Moor-Kreuzkraut-Honig.

\section{$\ddot{U}$ bersicht der einzelnen Honigproben}

Die Gruppe I umfasst sieben Frühjahrshonige, die in der Umgebung von Haarlem bemustert wurden und die vorwiegend (ausser Probe Nr. 6) aus einem Gebiet mit typischer Dünenvegetation stammen.

Die Honige Nr. 1 bis 5 kommen alle aus demselben verwilderten Garten. 
Nach imkerlichen Angaben handelt es sich bei Honig Nr. 1 um Rosskastanien-Honig. Es wurden $36 \%$ Aesculuspollen gefunden. Honig Nr. 5 wurde zwischen Rosskastanien- und Lindentracht geschleudert; im Pollenbild war Cynoglossum mit $78 \%$ vertreten, wobei gesagt werden muss, dass es sich, ebenso wie bei Honig Nr. 1 bis 4, um einen pollenarmen Honig handelte.

Honig Nr. 6. kam aus einem Obstgarten und wies $50 \%$ Prunuspollen auf.

Die Gruppe II umfasst sieben “ Lindenblütenhonige », geschleudert nach dem Blühen der Linden; sie stammen hauptsächlich aus verwilderten Gärten im Dünengebiet (Nr. 1-5).

Die Honigproben Nr. 6. und 7 wurden uns von Imkern aus dem östlichen Teil der Niederlande überlassen.

Um einen Eindruck des Tiliapollen-Anteils in niederländischen Lindenblütenhonigen zu bekommen, wurde die Häufigkeit des Tiliapollens in Prozenten ausgedrückt. Für die Honigproben Nr. 1-7 wurde beziehungsweise gefunden : 16, 6, 4, 8, 21, 22 und $3 \%$. Honig Nr. 1 enthält daneben noch $50 \%$ Trifolium repens-Pollen. Bemerkenswert ist das Vorkommen von Ligustrumpollen als Leitpollen im Sediment der Honigproben Nr. 3 und 4. In beiden Fällen hatten die Bienenkästen als Standort einen Garten mit Ligustrum. Honig Nr. 7 enthielt nur 3\% Tiliapollen; daneben waren beträchtliche Mengen von Sinapis arvensis-Pollen vorhanden. Die Bezeichnung " Lindenblütenhonig 》 für diesen Honig ist zweifelhaft, da der Sinnenbefund nicht auf sortenreinen Lindenblütenhonig hinweist : das charakteristische Minzenaroma ist nicht vorhanden. Demgegenüber weist Honig Nr. 3 mit $4 \%$ Tiliapollen ein sehr deutliches Minzenaroma auf. Die absolute Zahl der Pollen beträgt für diesen Honig 84.400 und für Honig Nr. 247.220 pro $10 \mathrm{~g}$.

Von Gruppe III kommen die ersten drei Honigproben aus dem Dünengebiet, die Honige Nr. 4 bis 8 aus den Mittel- und Ost-Niederlanden und Honig Nr. 9 aus dem südlichen Teil der Niederlande. Bemerkenswert ist das Vorkommen von Artemisia als Leitpollen, und zwar bis $84 \%$ in Honig Nr. 1. Der Geschmack dieses Honigs war deutlich bitter.

In den meisten Präparaten wurden Ligustrum und Cynoglossum gefunden; von der letztgenannten Pollenart war in Honig Nr. 5 sogar $55 \%$ vorhanden. Alle Proben wiesen im mikroskopischen Bild Trifolium repens und Tilia auf, wobei der höchste Trifolium repens-Anteil $44 \%$ betrug (Honig Nr. 4); es gab also keinen sortenreinen Kleehonig in dieser Gruppe. Die absolute Zahl der Pollen wurde bestimmt in den Honigproben Nr. 2 und 7; gefunden wurde $35.100 \mathrm{bzw} 34.000$ pro $10 \mathrm{~g}$ Honig.

Der holländische Heidehonig (Gruppe IV) wurde früher, als es noch ausgedehnte Heidegebiete gab, in den Niederlanden hoch geschätzt. Zur Zeit ist die Heidehonigproduktion aus eigenem Land nicht mehr sehr gross, was auf das Verschwinden der Heidegebiete zurückzuführen ist. Heute wird der 
TAB. 1. - Liste der Pollenformen, die in 41 holländischen Honigen gefunden wurden, mit Angabe der Häufigkeit.

TABL. 1. - Liste des pollens trouvés dans 41 miels hollandais, avec leur fréquence.

L : Leitpollen (ïber $45 \%$ ausgezählten Pollen)

B : Begleitpollen (16-45\%)

$\mathrm{L}:$ Pollen dominant (plus de $45 \%$ des pollens dénombrés) B : Pollen d'accompagnement (16-45\%) s : wichtiger Einzelpollen (3-15\%)

v : Einzelpollen (unter $3 \%$ )

$s$ : pollen isolé important (3-15\%) $\mathrm{v}$ : pollen isole (moins de $3 \%$ )

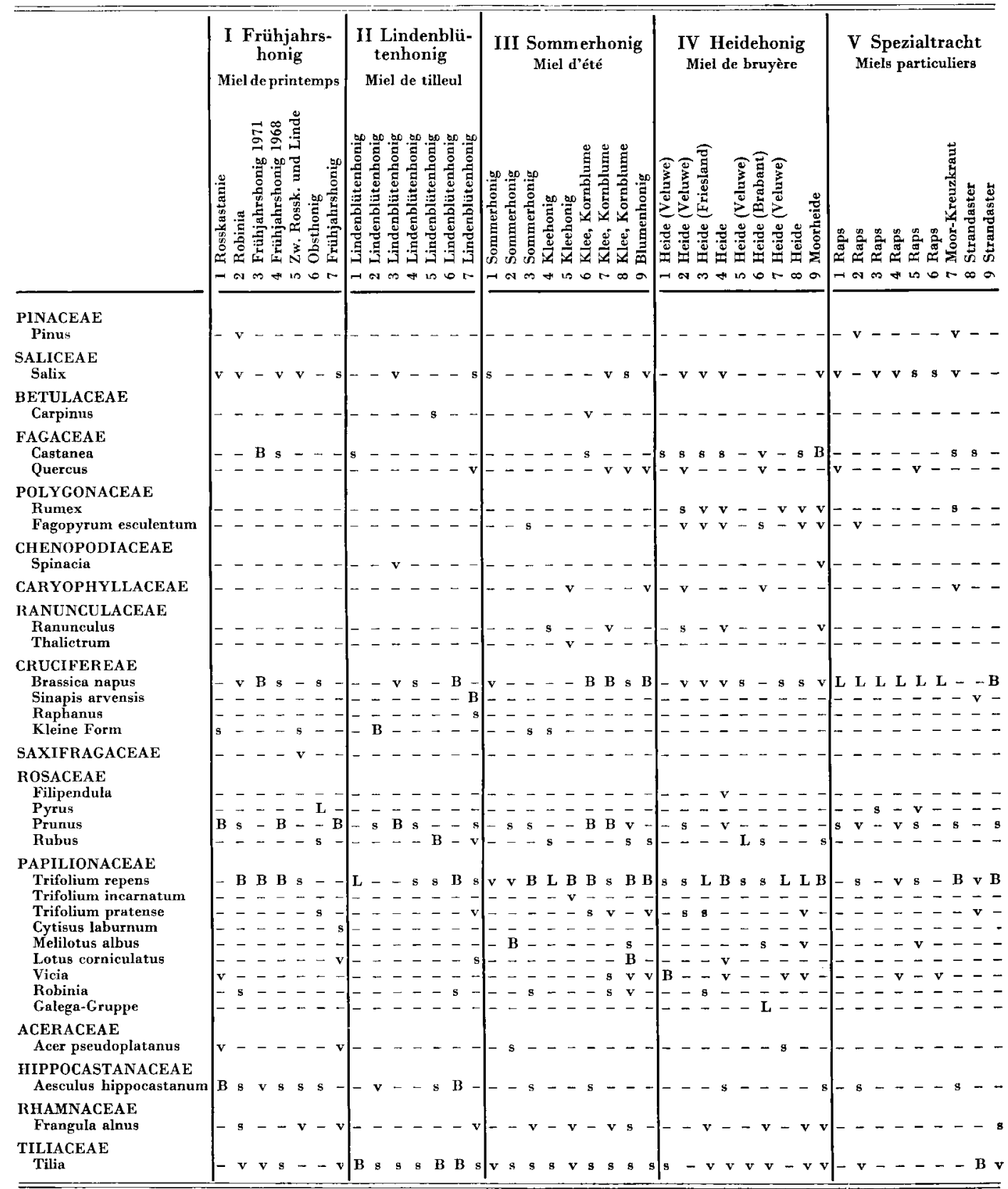


J. D. KERKVLIET, A.P.J. VAN DER PUTTEN

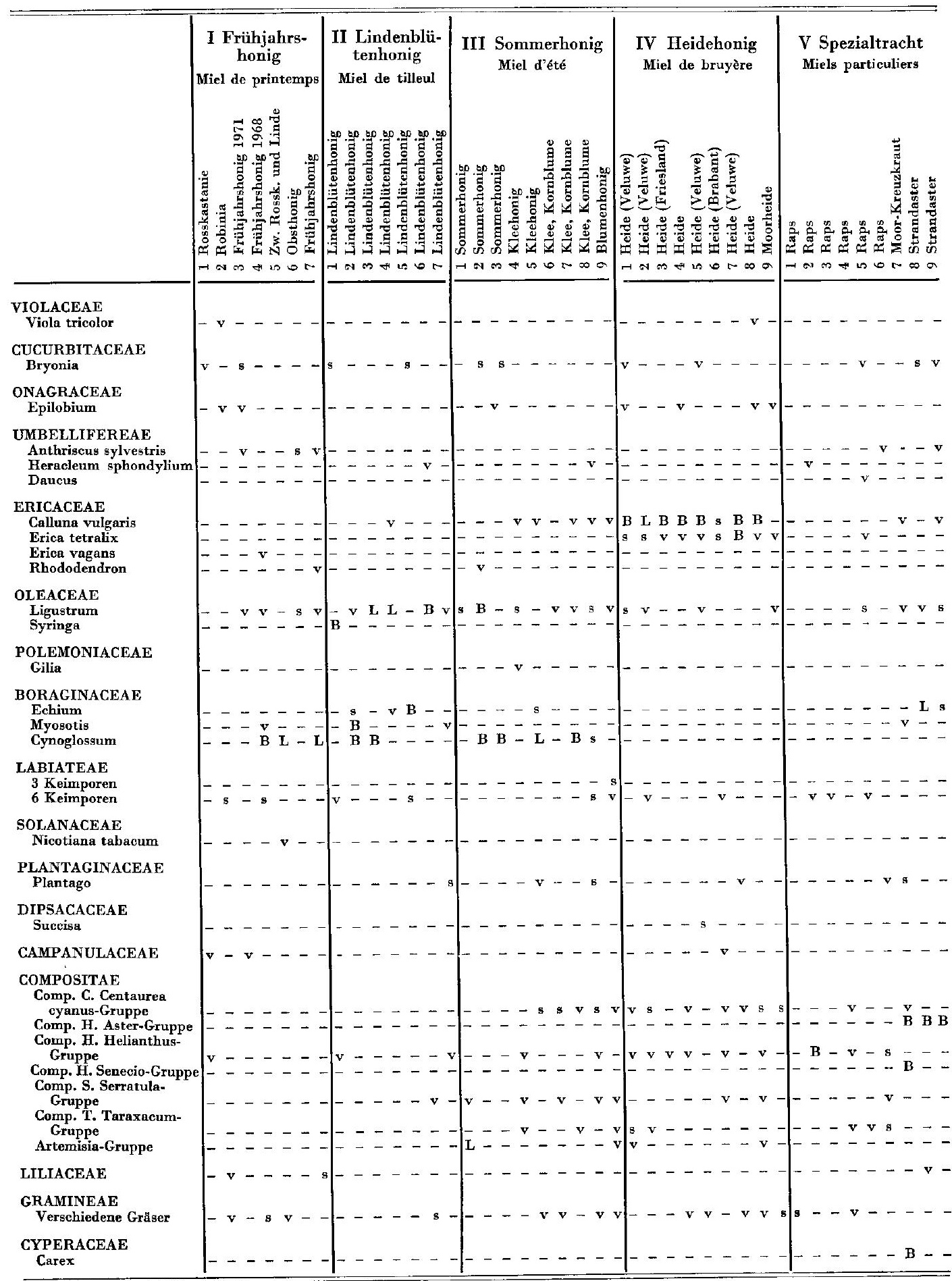


Heidehonig hauptsächlich importiert. Es gibt jedoch importierten Heidehonig, auf dem die Bezeichnung « Ausländischer Heidehonig » fehlt.

Daher ist es wichtig, das Pollensediment der niederländischen Heidehonige eingehend zu untersuchen.

Insgesamt wurden neun Heidehonige untersucht. Es handelte sich um Honig von Calluna vulgaris (Nr. 1 bis 8) und um eine Probe Moorheidehonig (Nr. 9). Die Herkunft der Honige Nr. 1, 2, 4 und 7 ist das Mittelniederländische Heidegebiet (Veluwe); Honig Nr. 3 wurde uns von einem Imker aus Friesland (nördlicher Teil der Niederlande) und Honig Nr. 6 von einem Imker aus Noord-Brabant (südlicher Teil der Niederlande) zur Verfügung gestellt. Honig Nr. 8 stammt aus Zuid-Limburg, dem südlichsten Teil der Niederlande.

Heidehonig kann wegen seiner zäh flüssigen Beschaffenheit nicht geschleudert werden; die Waben müssen gepresst oder gestippt werden. Der CallunaAnteil im Pollensediment gibt daher keine richtige Auskunft über den CallunaNektaranteil. Louveaux hat den Thixotropie-Test zur Charakterisierung des Heidehonigs durchgeführt (Louveaux 1966 und 1968).

Der Honig soll durch 12-stündige Erhitzung bei $65^{\circ} \mathrm{C}$ mit nachfolgender 24-stündiger Ruhezeit, nachdem der Honig zuvor in ein Röhrchen von $15 \mathrm{~mm}$ Durchmesser zentrifugiert wurde, völlig gelieren. Die Resultate des ThixotropieTestes sind in Tabelle 2 erwähnt, zusammen mit dem prozentualen Anteil der Calluna-und Erica-Pollen. Der Thixotropie-Test der Honigproben Nr. 2, 3, 4 und 7 fiel negativ aus. Sie waren offensichtlich mit einem anderen Honig gemischt worden (Honig Nr. 7 mit Moorheidehonig).

TAB. 2. - Thixotropie-Test, Zahl der Pollenkörner und prozentualer Anteil der Calluna- und Erica-Pollen in einigen niederländischen Heidehonigen.

TABL. 2. - Test de thixotropie, nombre de grains de pollen et pourcentage de pollen de Calluna et d'Erica dans quelques miels de bruyère néerlandais.

\begin{tabular}{|c|c|c|c|c|}
\hline \multirow{2}{*}{$\begin{array}{c}\text { Honigprobe } \\
\text { Echantillon de miel }\end{array}$} & \multirow{2}{*}{$\begin{array}{l}\text { Thixotropie-Test } \\
\text { Test de thixotropic }\end{array}$} & \multicolumn{2}{|c|}{$\begin{array}{c}\text { Prozentualer Anteil } \\
\text { Pourcentage }\end{array}$} & \multirow{2}{*}{$\begin{array}{c}\text { Zahl der } \\
\text { Pollenkörner } \\
\text { in } 10 \mathrm{~g} \text { Honig } \\
\text { Nombre de grains } \\
\text { de pollen dans } \\
10 \mathrm{~g} \text { de miel }\end{array}$} \\
\hline & & $\begin{array}{l}\text { Calluna } \\
\text { vulgaris }\end{array}$ & $\begin{array}{c}\text { Erica } \\
\text { tetralix }\end{array}$ & \\
\hline Nr. IV 1 & positiv & $23 \%$ & $5 \%$ & \\
\hline 2 & negativ & 43 & 5 & 47500 \\
\hline 3 & negativ & 18 & 2 & \\
\hline 4 & negativ & 40 & 2 & \\
\hline 5 & positiv & 32 & 3 & 144700 \\
\hline 6 & positiv & 7 & 7 & \\
\hline 7 & negativ & 20 & 25 & \\
\hline 8 & positiv & 16 & 2 & \\
\hline 9 & negativ & - & 2 & \\
\hline
\end{tabular}


Bemerkenswert ist das Auftreten von Castanea in niederländischem Heidehonig. Auch Jansen fand, in einer Untersuchung von 15 Heidehonigproben aus einem Heidegebiet südlich von Amsterdam, einen hohen Castanea-Anteil (Jansen en Pleuger 1973). Mommers fand in einer Probe niederländischen Heidehonigs sogar $79 \%$ Castanea mit $10 \%$ Calluna (Moммers 1968/1969). Uebrigens stimmen unsere Resultate mit den Untersuchungen überein, die Maurizio an holländischen Heidehonigen durchgeführt hat (Madrizıo 1966 und 1973).

Die fünfte Gruppe umfasst einige Spezialtrachten und zwar zum Teil aus unserem Flevopolder (neu angelegtem Polder). Die Honige Nr. 1 bis 6 sind Rapshonige, mit einem Brassica napus-Anteil von 96, 58, 93, 93, 86 und $75 \%$. Honig Nr. 7 ist ein Moor-Kreuzkraut Honig (Senecio paluster) mit $15 \%$ Senecio paluster-Pollen (Abbildung 1), ein grob kristallisierter, sehr dunkler Honig. Nr. 8 und 9 sind Strandasterhonige. Aster tripolium (Abbildung 2) trat darin als Begleitpollen auf, mit einem Anteil von $17 \mathrm{bzw} 30 \%$. Nach dem Vorkommen von Tilia als Begleitpollen im Strandasterhonig Nr 8 scheint es, dass dieser Honig vermischt worden ist.

MaUrizio (1959-1960) schreibt, dass Strandasterhonige einen deutlich salzigen Geschmack haben können.

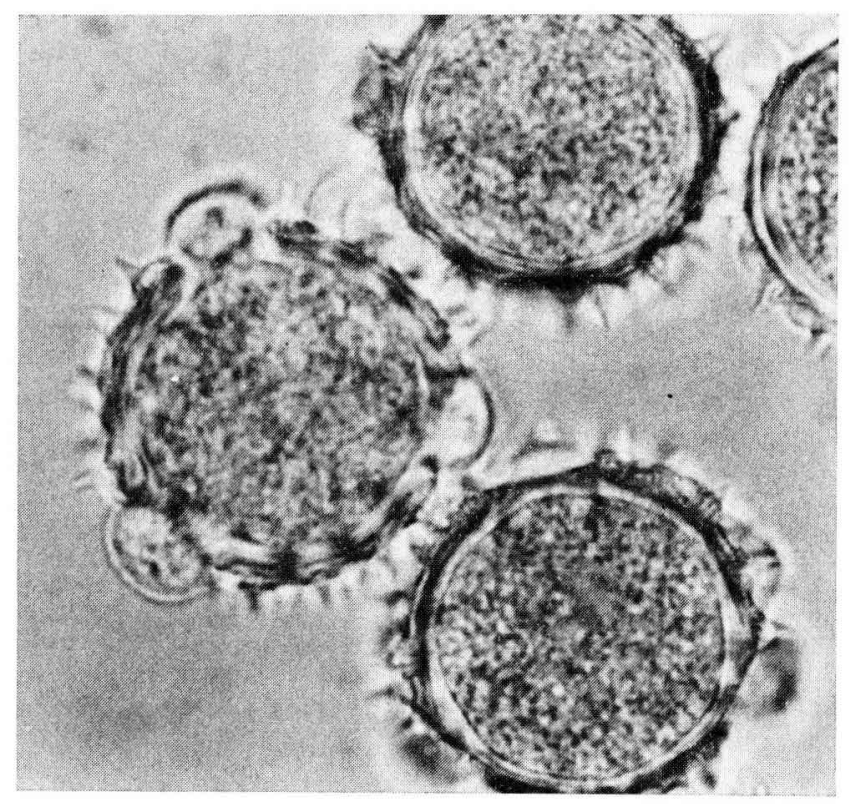

Aв8. 1. - Pollen von Senecio paluster Vergrösserung 1200-fach, Ölimmersion

Frg. 1. - Pollen de Senecio paluster Grossissement $1200 \mathrm{x}$, immersion 
In den vorliegenden zwei Proben konnte dies nicht konstatiert werden; ebenso wiesen sie keinen erhöhten Na-oder K-Gehalt auf.

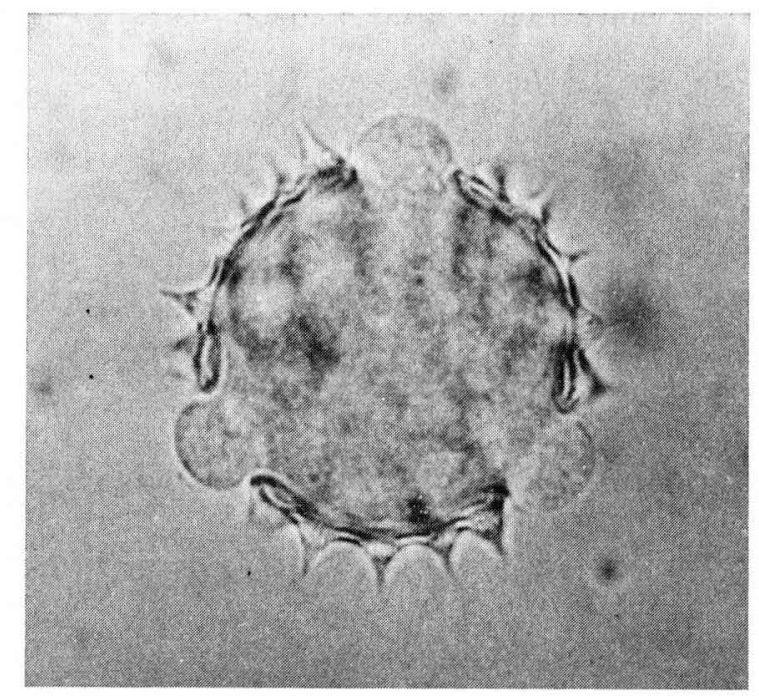

Авв. 2. - Pollen von Aster tripolium Vergrösserung 1200-fach, Ölimmersion

Fig. 2. - Pollen d'Aster tripolium Grossissement $1200 \mathrm{x}$, immersion

\section{DISKUSSION}

In den 41 niederländischen Honigen konnten insgesamt 65 verschiedene Pollenformen bestimmt werden. Die Häufigkeit, mit der einige dieser Formen in den untersuchten Honigen vorkommen, ist in Tabelle 3 angegeben.

Vor allem kommt Trifolium repens (Weissklee) als Trachtquelle in Betracht. In $80 \%$ der untersuchten Honigproben wurden Weisskleepollen gefunden. De Boer (1957) fand bei einer Untersuchung von 268 Honigen in 95,8\% der Proben Trifolium repens. An zweiter Stelle stehen bei ihm die Kreuzblütler (Crucifereae) mit 56,3\%. Sie kommen in unserer Arbeit an dritter Stelle $(63 \%)$. Bemerkenswert ist, dass Ligustrum an fünfter Stelle steht.

Verhältnismässig viel kommt Bryonia-Pollen vor $(27 \%)$, was darauf zurückzuführen ist, dass rund ein Drittel der untersuchten Proben aus dem Dünengebiet stammt, wo Bryonia sehr verbreitet ist.

Dagegen fand sich Frangula alnus vorwiegend in Honigen aus den Mittelund Ost-Niederlanden.

Centaurea cyanus wurde vorwiegend in Honigproben gefunden, die aus den 
östlichen Niederlanden stammten. Die zur Pflanzenfamilie der Boraginaceae gehörenden Gattungen Cynoglossum und Echium wurden vorwiegend in Honigen aus dem Dünengebiet gefunden. Zum Schluss sei auf das ziemlich häufige Vorkommen von Castanea in niederländischem Honig, besonders im Heidehonig hingewiesen.

Tав. 3. - Vorkommen einiger Pollenformen in den untersuchten niederländischen Honigproben.

TABL. 3. - Présence de quelques pollens dans les échantillons de miel néerlandais analysés.

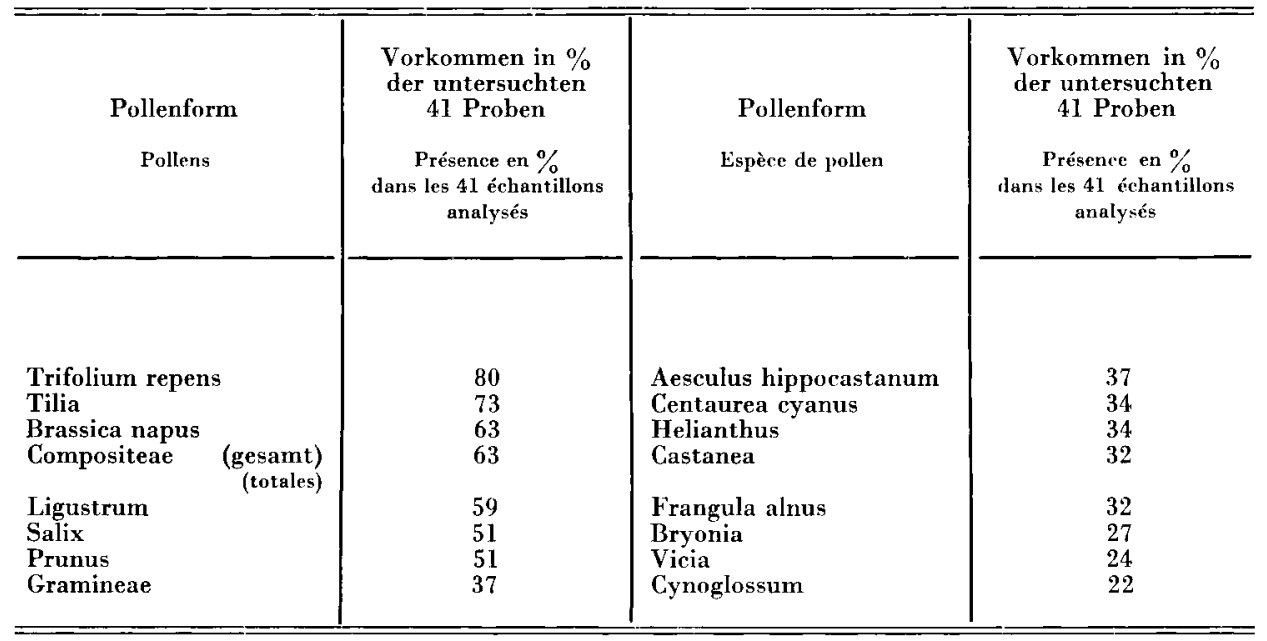

Zusammenfassend kann gesagt werden, dass in niederländischen Honigen vorwiegend Trifolium repens auftritt, in Kombination mit anderen Pollenformen. Besonders häufig sind die Kombinationen mit Tilia, Brassica napus, Compositeae, Ligustrum, Salix und Prunus.

Für niederländischen Heidehonig ist die Kombination Trifolium repens, Calluna vulgaris (Leit-oder Begleitpollen), Fagopyrum esculentum (Einzelpollen), Castanea (wichtiger Einzelpollen), Erica tetralix, Centaurea cyanus und Helianthus (Einzelpollen) charakteristisch.

'Eingegangen im Februar 1975

Reçu pour publication en février 1975

\section{DANKSAGUNG}

An dieser Stelle danken wir Herrn Dr. G. Vorwohl, Landesanstalt für Bienenkunde Stuttgart-Hohenheim BRD, für die Identifizierung einer unbekannten Pollenform.

Herzlich danke ich dem Europarat in Strassburg für die Gewährung eines Stipendiums, wodurch es möglich wurde, einige praktische Arbeiten auf dem Gebiet der mikroskopischen Honiguntersuchung bei Dr. G. Vorwohl, Stuttgart-Hohenheim, und Fr. Dr. A. Maurizio, Bern-Liebefeld, zu machen. 


\section{RESUMÉ}

On a étudié au microscope la présence de pollen dans un certain nombre d'échantillons de miel, d'origine néerlandaise certaine, aimablement fournis par divers apiculteurs, a fin de se faire une idée générale du spectre pollinique des miels néerlandais.

Par ailleurs on a examiné dans quelle mesure les dénominations florales établies d'après les observations des apiculteurs concordaient avec l'analyse microscopique.

A ce propos on a déterminé le pourcentage de pollen de Tilia (tilleul) dans les échantillons dénommés miels de tilleul par les apiculteurs. De même pour les miels de bruyère, on a procédé à la recherche de la teneur en pollen de Calluna (callune) et d'Erica (bruyère) et au test de thixotropie selon LoUveaux.

On a examiné, en outre, dans quelle mesure le spectre pollinique concordait avec les recherches effectuées antérieurement par DE BOER sur les miels néerlandais.

On peut dire en résumé qu'on rencontre Trifolium repens (trè fle blanc) dans les miels néerlandais en combinaison avec Tilia (tilleul), Brassica napus (colza), Compositeae (Composées), Ligustrum (troène), Salix (saule) et Prunus (prunier).

Pour le miel de bruyère néerlandais la combinaison Trifolium repens (trè fle blanc), Calluna vulgaris (callune), Fagopyrum esculentum (sarrazin), Castanea (châtaignier), Erica tetralix (bruyère à quatre angles), Centaurea cyanus (blenet) et Helianthus (tournesol) est caractéristique.

\section{LITERATURVERZEICHNIS}

DE BoEr H. W., 1957. - De samenstelling en de eigenschappen van in Nederland gewonnen honing. Maandschrift voor Bijenteelt 1957, 3-26.

Evgler A., 1964. - Syllabus der Pflanzenfamilien. Bornträger, Berlin-Nikolassee.

Jansen J.-T. en Pleuger J.-C., 1973. - Pollenanalyse van inlandse honingen. Rapport Keuringsdienst van Waren, Amsterdam.

Kerkvliet J.-D. en Van Der Putten A. P. J., 1973. - Pollenanalyse van nederlandse honing, De Warenchemicus 3 (3), 68-76.

Louveaux J., 1966. — Essai de caractérisation des miels de Callune. Ann. Abeille 9 (4), 351-358.

Louveaux J., 1968. - Die französischen Heidehonige. Z. Bienenforschung 9 (5), 211-215.

Louveaux J., Mavrizio A. und Vorwohl G., 1970. - Methodik der Melissopalynologie. Apidologie 1 (2), 193-209.

Maurizio A., 1959-1960. - Blüte, Nektar, Pollen, Honig. Deutsche Bienenwirtschaft 1959$1960,1-48$.

Maurizio A., 1966. — Das Pollenbild europäischer Heidehonige. Ann. Abeille 9 (4), 375-387.

Maurizio A., 1973. - The heather honeys of Europe, Bee World 54 (3), 111-116.

Mommers Mieke C. S., 1968-1969. - Onderzoek naar het voorkomen van coniferenhoning in Nederland. Maandschrift voor Bijenteelt 70 (12), 170-174 (1968) en 71 (1), 5-12 (1969). 